\title{
Improving Ground Penetrating Radar Imaging in High Loss Environments by Coordinated System Development, Data Processing, Numerical Modeling, and Visualization Methods with Applications to Site Characterization
}

EMSP Project 86992

Annual Report as of 6/2003

\section{Research Objective:}

The Department of Energy has identified the location and characterization of subsurface contaminants and the characterization of the subsurface as a priority need. Many DOE facilities are in need of subsurface imaging in the vadose and saturated zones. This includes 1) the detection and characterization of metal and concrete structures, 2) the characterization of waste pits (for both contents and integrity) and 3) mapping the complex geological/hydrological framework of the vadose and saturated zones. The DOE has identified ground penetrating radar (GPR) as a method that can non-invasively map transportation pathways and vadose zone heterogeneity. An advanced GPR system and advanced subsurface modeling, processing, imaging, and inversion techniques can be directly applied to several DOE science needs in more than one focus area and at many sites. Needs for enhanced subsurface imaging have been identified at Hanford, INEEL, SRS, ORNL, LLNL, SNL, LANL, and many other sites. In fact, needs for better subsurface imaging probably exist at all DOE sites. However, GPR performance is often inadequate due to increased attenuation and dispersion when soil conductivities are high.

Our objective is to extend the limits of performance of GPR by improvements to both hardware and numerical computation. The key features include 1) greater dynamic range through real time digitizing and receiver gain improvements, 2) modified, fully characterized antennas with current sensors to allow dynamic measurement of the changing radiated waveform, 3) modified deconvolution and depth migration algorithms exploiting the new antenna output information, 4) development of automatic full waveform inversion made possible by the known radiated pulse shape.

\section{Research Progress and Implications:}

This report summarized progress after 10 months of a 3 year project. A critical element in the research is to extend the effective depth of investigation by increasing the dynamic range of GPR. The progress we have made on this element includes:

a. Identification and procurement of the most suitable, highest performance, waveform digitizer/averager. We selected and procured an Acqiris model AP-200. This unit allows us to digitize and average waveforms in real time with no equivalent-time sampling needed. This yields a substantial improvement in signalto-noise gain by realtime waveform averaging.

b. LabView data acquisition software has been written for the AP-200. This software also includes a provision for including differential global positioning system data in the data stream. This provision is important because accurate positions are crucial to high quality GPR subsurface images, particularly 3D images.

c. Two alternative methods of electronic receiver dynamic ange extension have been investigated: 1) Real time gain ramping to preferentially boost the amplification of later 
(smaller) signals relative to the earlier (larger) signals, and 2) Linear/logarithmic amplification that linearly amplifies small signals, but progressively decreases the gain for larger signals. Both of these approaches show promise as shown in Figures 1 and 2.

Figure 2 demonstrates the linear/logarithmic amplifier at two different input signal levels.

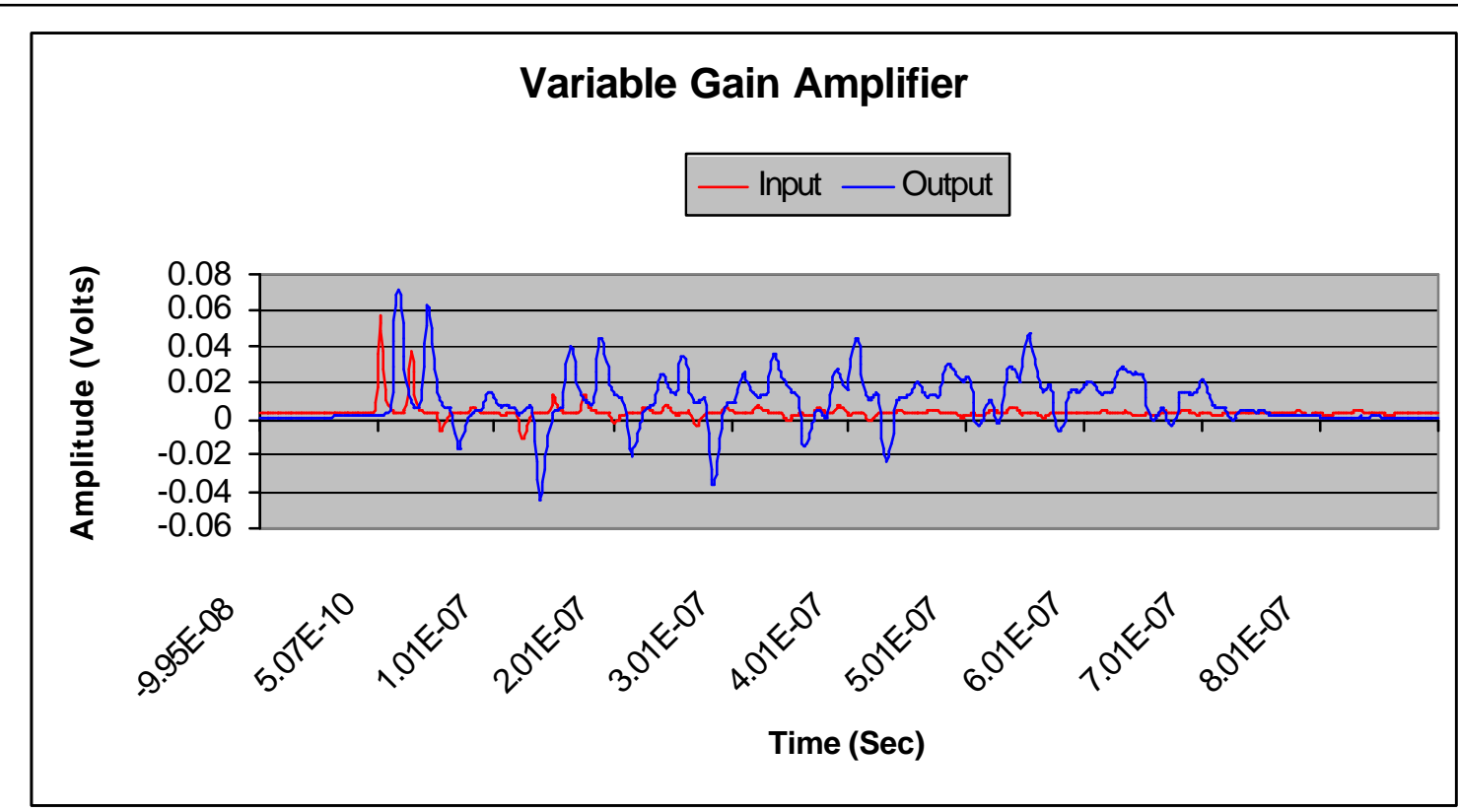

Figure 1. This figure shows an input waveform (red) into a time-varying gain amplifier and a typical output waveform (blue). The gain ramp was adjusted so that the amplifier keeps the amplitudes of early and late signals comparable, thus reducing the dynamic range requirement on the waveform digitizer.

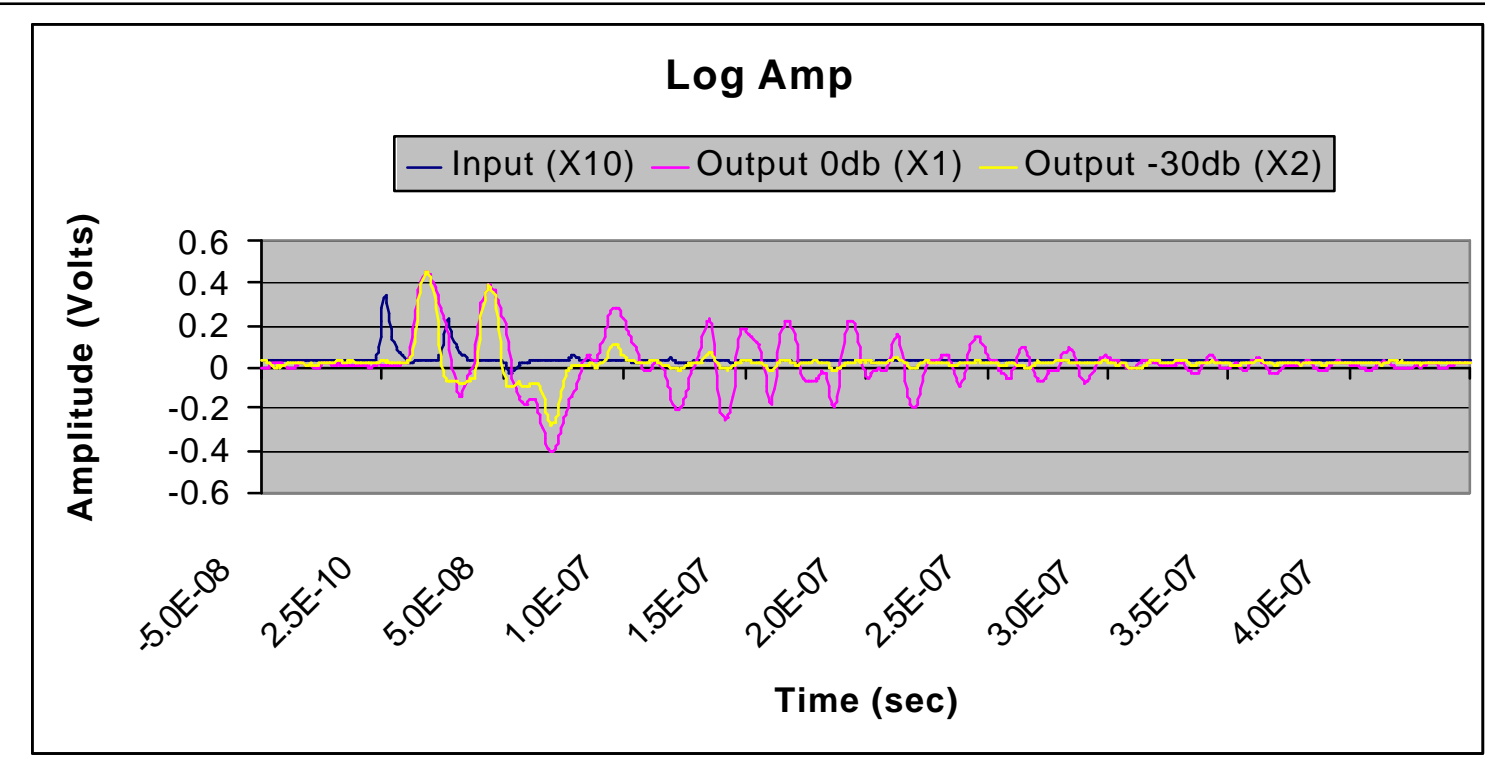

Figure 2. This figure shows an input train of diminishing amplitude pulses into a linear/logarithmic amplifier at two different input attenuator settings $(0$ and $-30 \mathrm{~dB})$. Small signals are highly amplified. Large signals are compressed. 
A second element is antenna design. We have numerically simulated the radiated waveforms from linear dipoles and resistively loaded linear dipoles and bowtie antennas with and without backshields and with a variety of driving pulse shapes and a range of soil conductivities. These continuing simulations, conducted using a finite difference time domain program, will guide our antenna designs and pulser design.

We have also begun numerical modeling to study the effects of soil conductivity on radiated waveform amplitudes and distortion due to dispersion (frequency dependent velocity) and the effect of errors in the assumed waveform on signal processing methods such as deconvolution, to remove the effect of the radiated waveform on the resulting image, and migration, to correct the effects of layer dipping and other geometrical effects on the image. Figure 3 shows the effect of changes in near surface soil relative dielectric permittivity and conductivity on the radiated waveform.

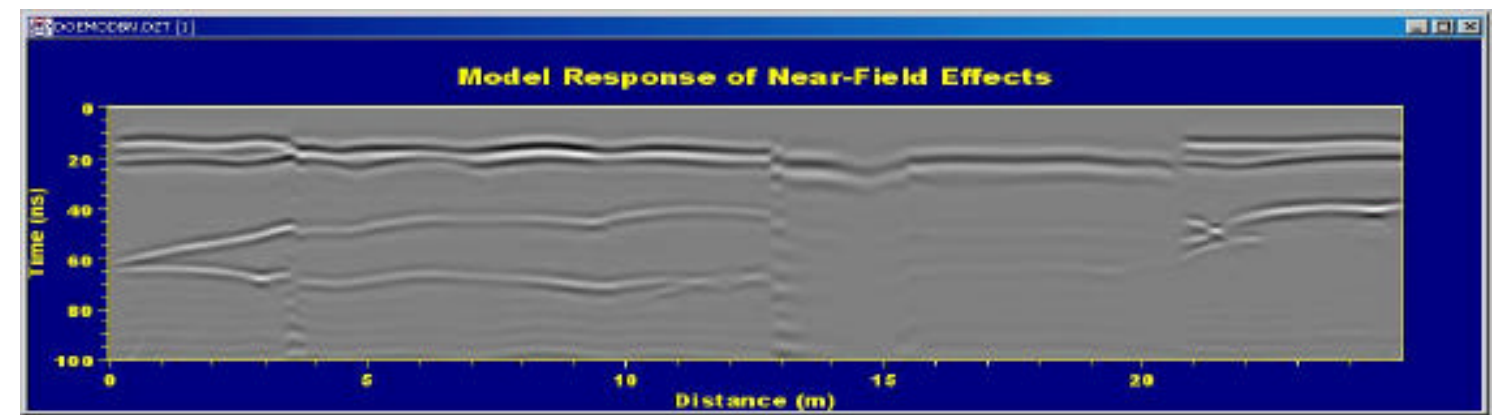

$-$

Model with Near-Field Variations

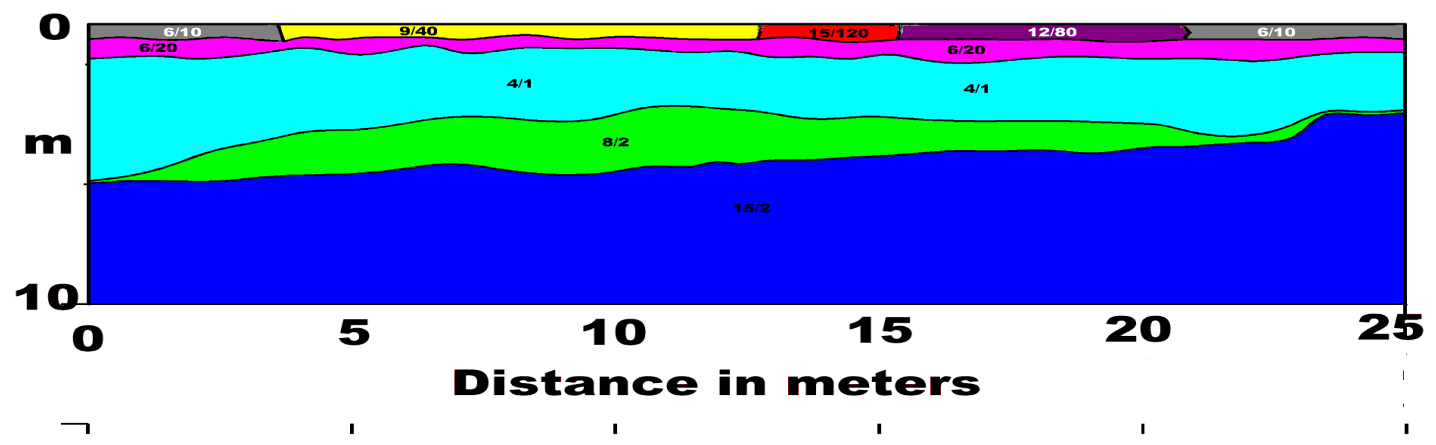

Figure 3. The top panel shows a simulated radar profile over the model shown in the lower panel. In the lower panel the soil relative dielectric permittivity is given by the first number in the pairs of numbers and the electrical conductivity in $\mathrm{mS} / \mathrm{m}$ is given by the second number. For the small thin section (red) with a high electrical conductivity of $120 \mathrm{mS} / \mathrm{m}$ the simulated GPR profile in the top panel shows slowing (late signal arrival), high attenuation (low signal amplitude), and dispersion (pulse broadening).

\section{Planned Activities:}

We plan to continue our antenna modeling studies, build physical antennas with current measuring elements included, implement one of our new receiver designs, build a new pulser for the antennas, test our antennas and systems and integrate all necessary physical elements into a fieldworthy prototype system by approximately June, 2004. In parallel 
with the development of the system we will work on the development of an automatic inversion to better estimate the properties of unknown layers by taking advantage of better knowledge of the actual radiated waveform from recorded transmitting antenna data. When a working prototype is available we will look for applications at DOE sites.

\section{Information Access:}

Further information on this project may be obtained from http://www.pnl.gov/emsp/fy2003/presentations/wright_david_86992.pdf. 